\title{
Design and optimization of Compact Linear Collider main linac accelerating structure
}

\author{
Hao Zha and Alexej Grudiev* \\ CERN, European Organization for Nuclear Research, Geneva 1211, Switzerland
}

(Received 6 July 2016; published 16 November 2016)

\begin{abstract}
The Compact Linear Collider (CLIC) main linac uses waveguide damped structure as its baseline design. The current baseline structure design written in the CLIC Conceptual Design Report is named "CLIC-G." Recent activities on the CLIC-G design including high power tests on structure prototypes and the study of machining cost assessment had raised the need of reoptimizing the structure design to minimize the machining cost and the pulse surface temperature rise. This work presents optimization of the structure geometry, high-order-mode (HOM) damping loads and the design of a HOM-free power splitter for the input coupler. Compared to the current baseline design CLIC-G, the new structure design reduced the pulse surface temperature rise, input power and manufacturing cost and achieves better suppression to the long range transverse wakefield. Cell disks and damping loads for the new structure design are also more compact than those of the CLIC-G design.
\end{abstract}

DOI: 10.1103/PhysRevAccelBeams.19.111003

\section{INTRODUCTION}

Waveguide damped structure is studied as the baseline accelerating structure design for the Compact Linear Collider (CLIC) main linac [1]. As shown in Fig. 1, a regular cell of the structure has four waveguides with damping loads inside to damp unwanted high order modes (HOMs) in order to preserve the beam stability in multiple bunch operation. These waveguides are dimensioned to cut off the fundamental working frequency in order to prevent the degradation of the fundamental mode $Q$-factor. The current structure design described in the CLIC Conceptual Design Report (CDR) [2] is named "CLIC-G." It operates at $11.994 \mathrm{GHz}$ in $2 \pi / 3$ modes at an accelerating gradient of $100 \mathrm{MV} / \mathrm{m}$. The one structure unit is about $250 \mathrm{~mm}$ long with 26 regular cells plus two compact couplers. The detailed parameters are introduced in [3]. Other structures with HOM suppression are also studied as alternative designs of the CLIC main linac [4,5].

Due to the importance of the long-range transverse wakefield suppression in the CLIC main linac, an experimental verification in this aspect of the CLIC-G design had been carried out in the FACET facility [6]. Figure 2 shows the measured results together with GDFIDL [7] simulations on the same structure [8]. The transverse wakefield measured at the position of the second bunch was $5 \mathrm{~V} / \mathrm{pC} / \mathrm{m} / \mathrm{mm}$, which verified the long-range wakefield suppression of the CLIC-G design. The excellent

\footnotetext{
*alexej.grudiev@cern.ch

Published by the American Physical Society under the terms of the Creative Commons Attribution 3.0 License. Further distribution of this work must maintain attribution to the author(s) and the published article's title, journal citation, and DOI.
}

agreement between simulations and measurements as demonstrated in the plots gives us confidence in the accuracy of the wakefield simulation codes. This is important for the design work described below where all improvements made on the structure and HOM damping load design are based on simulations.

High power tests of CLIC structures have been under way at several test stands at CERN and KEK [9-17]. The results are summarized in Fig. 3 [18]. Recent tests show that the unloaded gradient achieved in the CLIC-G prototype structure for the CLIC effective pulse length (180 ns) and required breakdown rate $\left(3 \times 10^{-7}\right.$ per meter structure per pulse) is $96 \mathrm{MV} / \mathrm{m}$. The corresponding loaded gradient would be about $80 \mathrm{MV} / \mathrm{m}$. The highest gradient demonstrated so far is $120 \mathrm{MV} / \mathrm{m}$ which was achieved in an undamped structure with 24 cells with an iris geometry identical to the CLIC-G design. Generally speaking, the gradients achieved in damped CLIC structures were lower than those of undamped ones. One possible explanation is

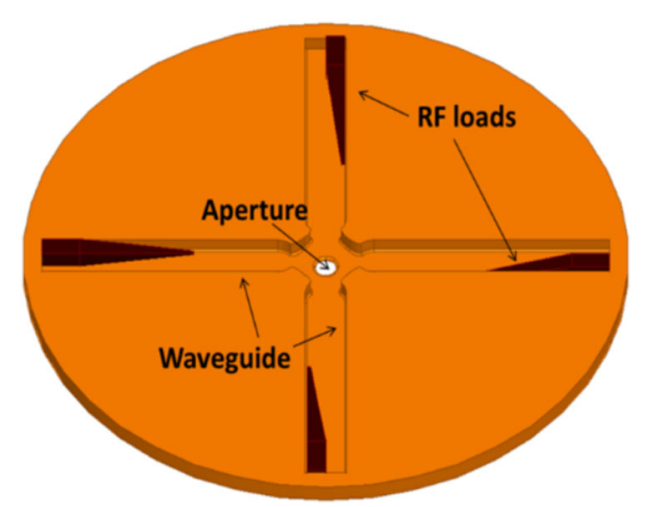

FIG. 1. One cell of waveguide damped structure. 


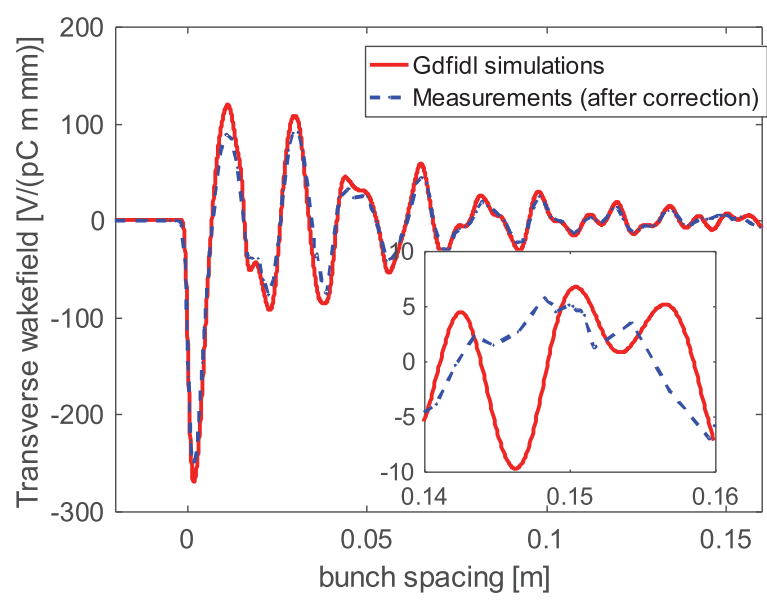

FIG. 2. Results of wakefield measurements.

that the pulse surface temperature rise of the damped structures is about $50 \mathrm{~K}, 2$ times higher than that of undamped structure for the $100 \mathrm{MV} / \mathrm{m}$ loaded gradient.

Assessment of the machining cost of the CLIC-G structure was also studied [19]. The cost model shows that a structure cell with the larger rounding will significantly reduce the manufacturing cost. Changing the rounding in the geometry will also have the impact on the structure design in the aspect of surface field and wakefield suppression, which results in a new structure design. This study, together with the high power tests for the CLIC-G structure, had raised the need for reoptimizing the structure geometry to minimize the machining cost and the surface magnetic field. The detailed work of optimizing the structure geometry is described in Secs. II and III.

In order to accomplish the new structure design with a full HOM damping scheme, the geometry of damping loads was optimized and details are introduced in Sec. IV.

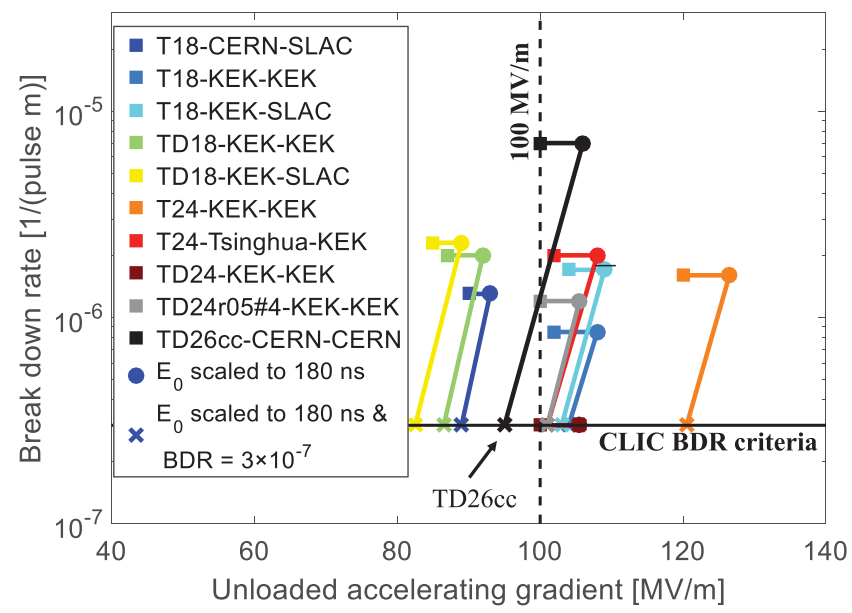

FIG. 3. High power tests on CLIC accelerating structure prototypes (the legend item is Name of structure-Place of fabrication-Place of high power test, BDR is the breakdown rate, $E_{0}$ is accelerating gradient) [18].

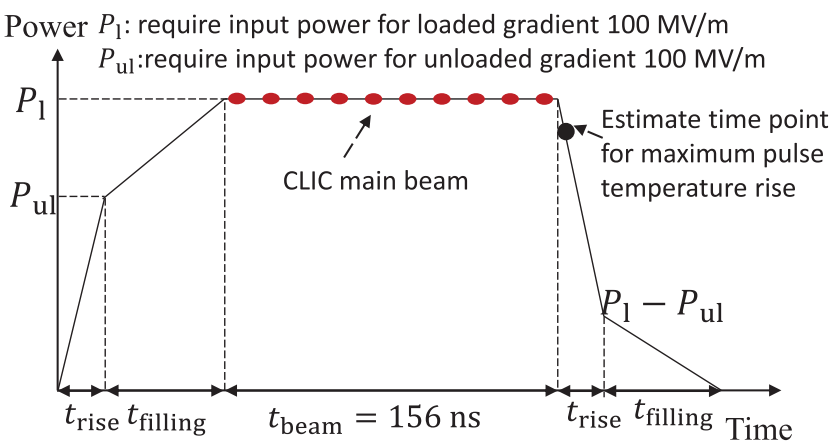

FIG. 4. Pulse surface heating calculation in CLIC accelerating structures.

A special rf power splitter based on a Magic-T geometry was designed to split the input power for the double feeding scheme as well as suppress the dipolar wakefield excited in the input coupler cell, which is introduced in Sec. V.

\section{GEOMETRY OPTIMIZATION OF WAVEGUIDE DAMPED CELLS}

\section{A. New wall geometry}

Pulsed surface heating in the accelerating structure is one of the main limitations for achieving high accelerating gradient [20-22]. It causes the damage to the wall surface, especially the bonding joint points. A scanning electron

(a)

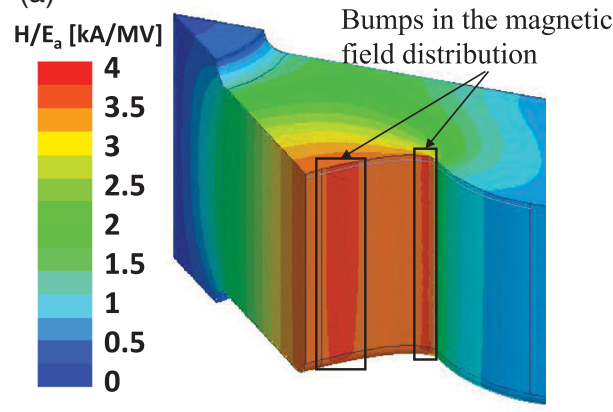

(b)

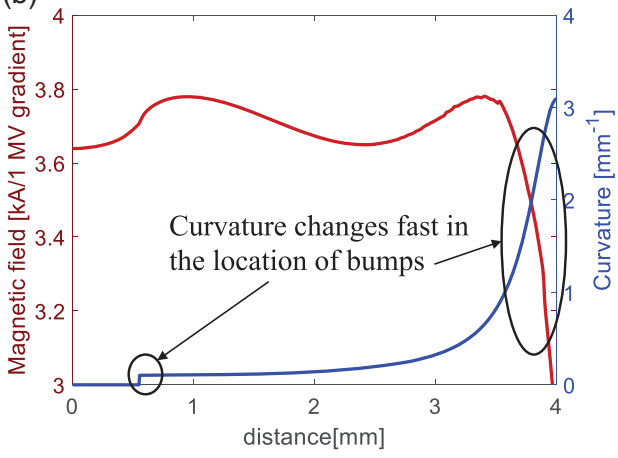

FIG. 5. (a) Magnetic field distribution in the middle cell of CLIC baseline accelerating structure (1/8 view); (b) curvature of the wall profile. 
microscope (SEM) was used to observe the breakdown points in the surface of CLIC-G structure cells [23]. Appearances of breakdown points showed a strong dependence on the surface pulse temperature rise according to SEM studies. Consequently, reducing the pulsed surface heating in the CLIC-G structure is one of the main objectives in this work.

The surface pulse heating is dependent on the material property, rf frequency, and the surface magnetic. The heating is accumulating in the rf pulse and the temperature rise is calculated by the rf pulse shape as described in [24] [page 98, Eq. (3-36)]. The input power pulse shape of the CLIC design is plotted in Fig. 4. Two ramping periods with different slope in the pulse shape is the dedicate design for beam loading compensation $[25,26]$. The maximum temperature rise is at the end of the flattop in the rf pulse, as seen in Fig. 4.

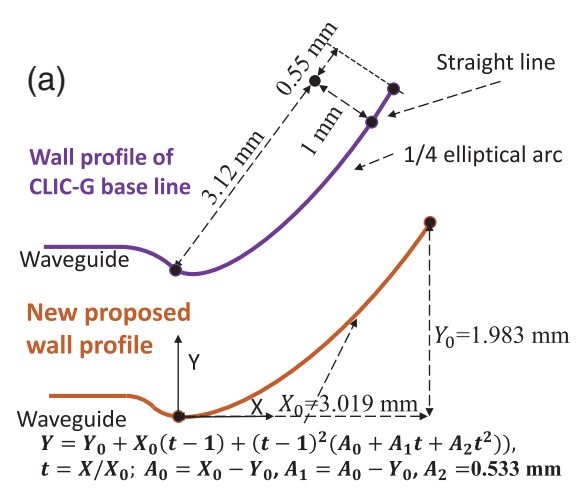

(b)
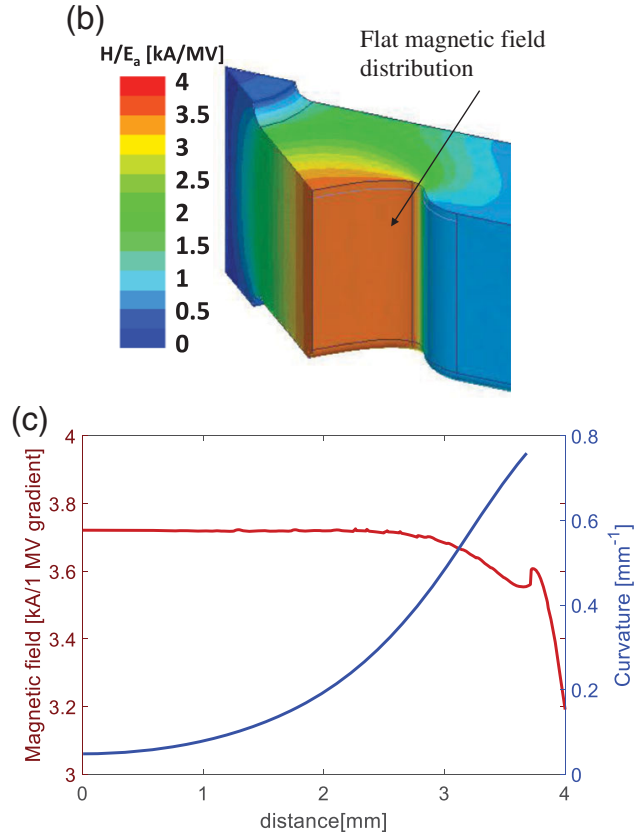

FIG. 6. (a) New proposed profile based on fourth order polynomial function (1/8 view). (b) Magnetic field distribution in the middle cell of CLIC accelerating structure with new proposed profile (1/8 view). (c) Curvature of the new proposed wall profile.
As shown in Fig. 5(a), the high magnetic field of the waveguide damped cell is concentrated at the cell wall. The profile of the cell wall in the CLIC-G design is a quarter of the elliptical arc plus a straight line, as seen in Fig. 6. This elliptical wall geometry had been optimized to have a flattened magnetic field distribution along the wall profile line to avoid the hot spot on the wall. However, two bumps can still be seen from the plot. The enhancement of magnetic field at the two bumps is due to the mutation of curvature in the profile line, as seen in Fig. 5(b).

The wall geometry could be improved and the bumps removed from the field distribution. A fourth order polynomial function based profile was proposed, as shown in Fig. 6(a). The shape of this proposed polynomial profile and the old elliptical one are roughly similar to each other. The main improvement of this polynomial profile is near the conjunction point of cavity and waveguide, where about $20 \mu \mathrm{m}$ discrepancy is presented between two curves (machining accuracy is about $2 \mu \mathrm{m}$ ). The polynomial curve has a smoother curvature change compared to the elliptical one as seen in Figs. 5(a) and 6(c). The magnetic field distribution of both wall profiles for the middle cell of the baseline design were simulated by a high frequency electromagnetic field calculation code (HFSS) [27] and are plotted in Fig. 6(b). The polynomial function based design decreases the maximum magnetic field by $2 \%$, which corresponds $\sim 2 \mathrm{~K}$ temperature reduction to the pulse surface heating.

\section{B. Rounding and waveguide geometry}

Milling is the main process in the machining of CLIC accelerating structures. An arc shape is usually employed in the milling head cutter in order to avoid a sharp edge and protect the cutter. During the milling process, the cutter leaves arc-shape ridges on the metal surface which introduce roughness on the structure surface (as seen in Fig. 7). The height of the ridge is determined by the milling step and the radius of the arc shape in the milling cutter, as expressed in the formula in Fig. 7. A small cutter radius requires a finer milling interval which consumes more machining time and cost, in order to meet the same surface roughness specifications.

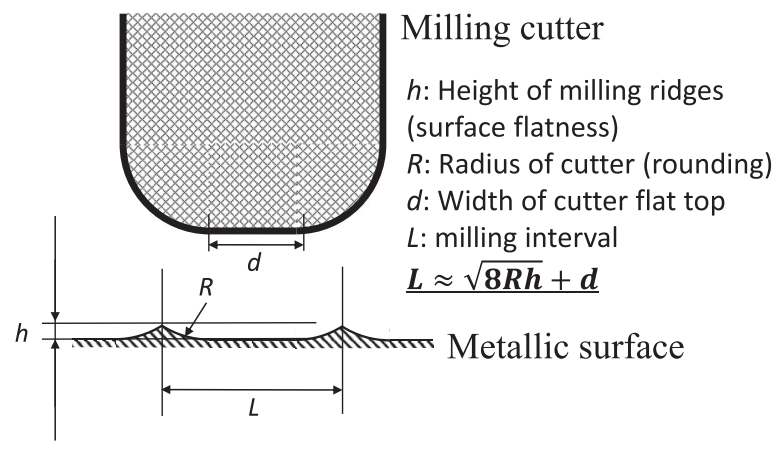

FIG. 7. Surface flatness and rounding in the milling process. 


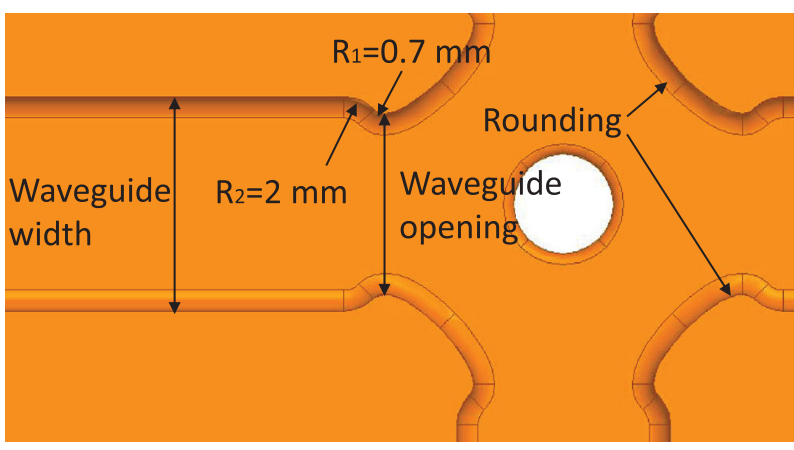

FIG. 8. Geometrical parameters in one structure cell related to the rounding study.

The cutter radius is limited by the minimum rounding in the structure as shown in Fig. 8. Thus increasing the rounding of the cell structure can reduce the machining cost. However, the larger rounding reduces the cross-section area of the waveguide and consequently the coupling of the HOM fields into the waveguides. In this case, damping waveguides with larger width and opening are needed for structure cells to maintain same wakefield suppression. However, increasing waveguide dimensions results in a higher surface magnetic field. Thus, a comprehensive study was necessary for the selection of the rounding.

Among all the geometrical parameters (rounding radius, waveguide width and waveguide opening) related to the rounding study, the width of the waveguide opening had the most significant impact on both the wakefield suppression and the surface magnetic field. The strategy in the optimization was to first determine the waveguide opening for different rounding and waveguide widths. When the waveguide opening is larger, the wakefield suppression goes stronger but the surface magnetic field goes higher. In order to minimize the surface magnetic field, the value of waveguide opening was as small as to keep the wakefield suppression the same level as the baseline CLIC-G design,

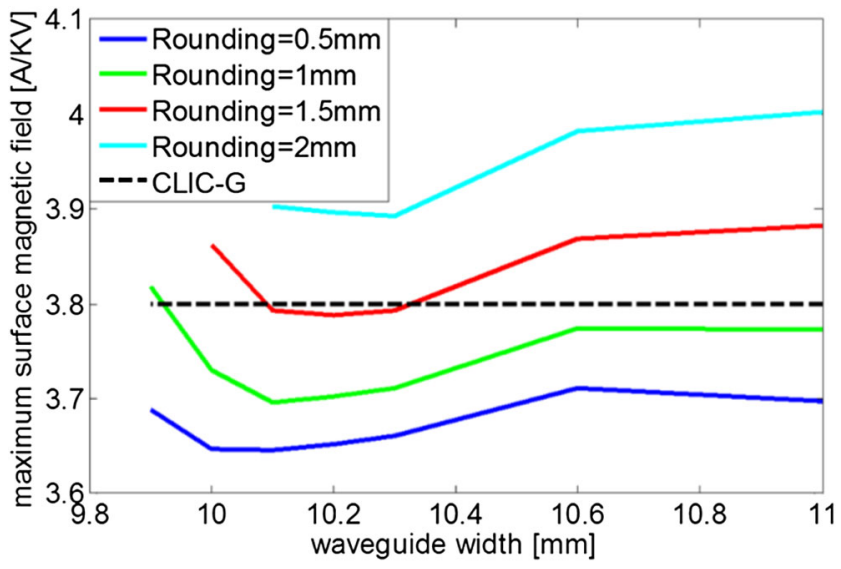

FIG. 9. Maximum magnetic field versus rounding and waveguide width.

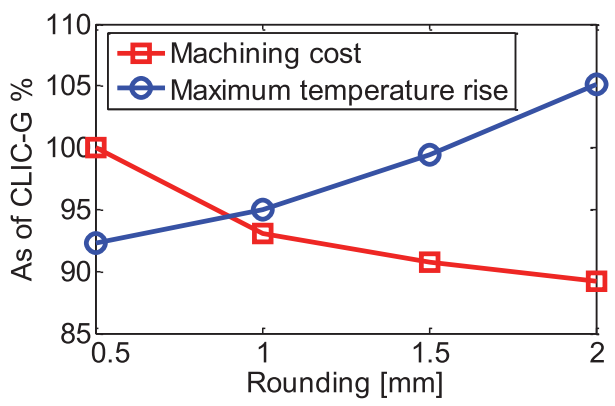

FIG. 10. Machining cost and pulsed surface heating versus rounding.

which is given by GDFIDL simulations of the long-range transverse wakefield in the tapered CLIC-G structure. With these minimum waveguide openings, HFSS simulations of the CLIC-G middle cell with different rounding and waveguide widths were carried out. The plot of maximum surface magnetic field versus rounding and waveguide width are shown in Fig. 9. As seen in the plots, the optimum waveguide width is around $10-10.3 \mathrm{~mm}$ for all roundings.

A plot of machining cost reduction [19] and reduction of the pulsed surface heating temperature rise versus rounding is shown in Fig. 10. The temperature rise was calculated from the solutions with optimum waveguide width. Due to the optimization of the wall profile and waveguide width, the temperature rise of the solution with $0.5 \mathrm{~mm}$ rounding is lower than that of CLIC-G, leaving margin to increase the rounding. We chose the $1 \mathrm{~mm}$ rounding design (corresponding optimum waveguide width: $10.1 \mathrm{~mm}$ ) for its significant reduction both on cost and temperature rise.

\section{FULL TAPERED STRUCTURE DESIGN}

\section{A. From single cell to the tapered structure}

The baseline CLIC-G design has 26 regular cells and two compact coupler cells as shown in Fig. 11. The iris apertures in the design range from 3.15 to $2.35 \mathrm{~mm}$. The average value and tapering of the iris aperture was determined by the global optimization [28] which considered the short range wakefield [29], shunt impedance, etc.

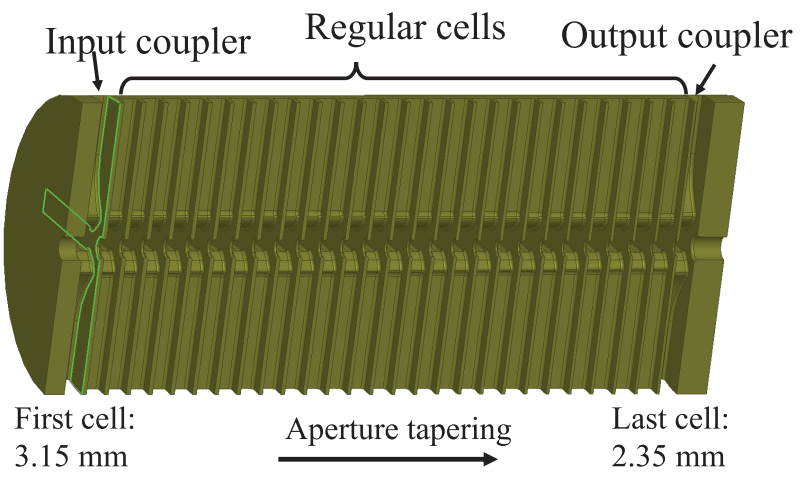

FIG. 11. Sketch of CLIC accelerating structure. 
The iris profiles have been optimized to minimize the surface electric field and the modified Poynting vector Sc [30]. This iris geometry is already well designed and will not be changed in the new design.

The optimizations introduced in Sec. II were for the middle cell of the CLIC structure. These optimizations should be extended to all other cells in order to get the full tapered structure design. Dimensions in the wall profile were individually optimized for each cell to get flattened distributions of magnetic field. The optimization of rounding $(1 \mathrm{~mm})$, waveguide width $(10.1 \mathrm{~mm})$ and waveguide openings $(8.14 \mathrm{~mm})$ were the same for all the cells.

In the baseline CLIC-G design, the upstream cell has the highest point for the pulse temperature rises, as seen in Ref. [3]. Due to the dependency of the surface magnetic field on the waveguide opening width, the temperature rise of upstream cells could be decreased by reducing the waveguide openings. Meanwhile, the waveguide openings of downstream cells were increased to compensate the weakening of wakefield suppression in upstream cells. Figure 12 shows the tapering on the waveguide openings versus the change of temperature rises. A quadratic function based tapering on the waveguide openings was selected and the maximum temperature rise decreases by $2 \mathrm{~K}$.

\section{B. Design of input and output couplers}

The baseline CLIC-G design uses similar geometry of regular cells in the design of the input and output couplers, as shown in Fig. 13. This is called compact coupler design which can also provide accelerating field and increase the active acceleration length. Two of the waveguide branches
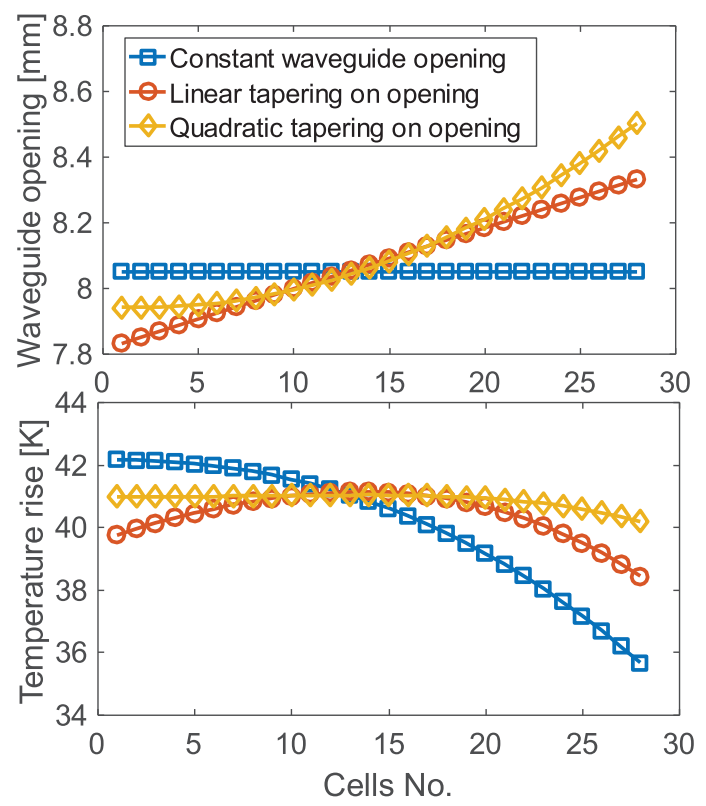

FIG. 12. Tapering on waveguide openings and corresponding temperature rise distributions.

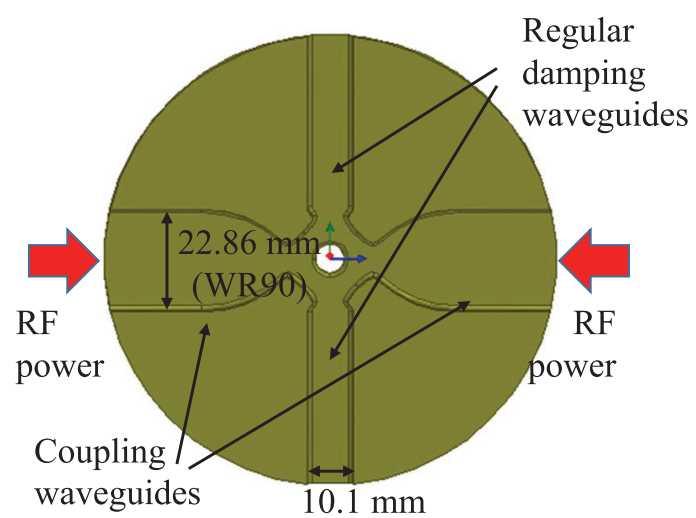

FIG. 13. Geometry of compact coupler cell.

(damping waveguides) in compact coupler cells use the same geometry as that of the nearest regular cell: dimensions of damping waveguides in the input coupler cell were taken from the first regular cell; and dimensions of the output cell were from the last regular cell. The other two waveguide branches (coupling waveguides) in coupler cells have larger waveguide widths (WR90 dimension) in order to transmit the fundamental $\mathrm{rf}$ power.

The dimensions of the waveguide openings in the coupling waveguides are adjusted to match the coupling from WR90 waveguides to structure cells. The design procedure was to match the output coupler first, otherwise the reflection at the output coupler will disturb the tuning and matching the upstream of the structure including the input coupler. Reflection due to the mismatch in the output coupler will cause a standing wave component in the electrical field distribution of structure cells. As seen in Fig. 14, the backward reflection at the output coupler is not the same as the port reflection coefficiency $S_{22}$ due to both the structure tapering and the finite conductivity of the structure wall. This backward reflection could be only calculated by the amplitude of frontward and backward wave analyzed from the field distribution of downstream cells, as expressed in the equation below:

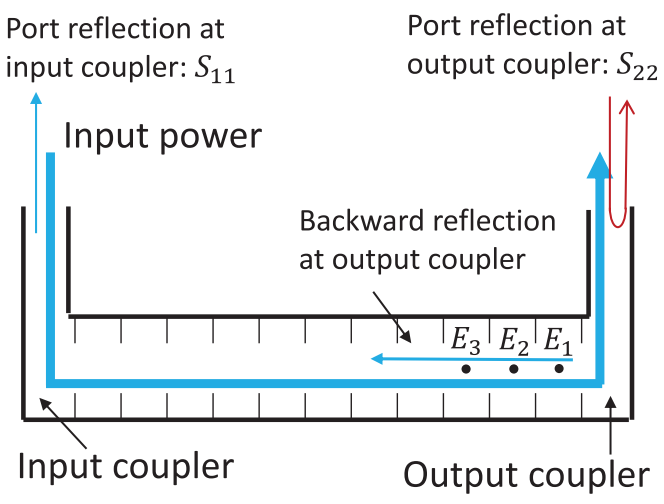

FIG. 14. Reflection in the output coupler. 


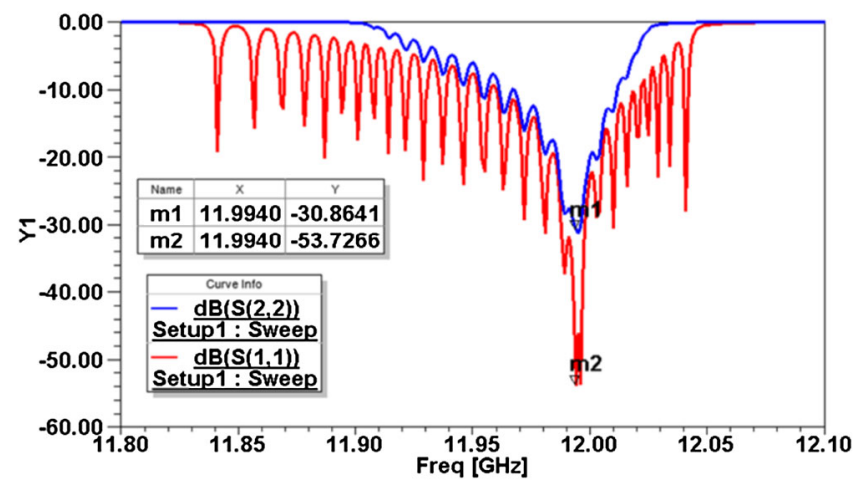

FIG. 15. Port-reflection of new CLIC-G tapered structure (HFSS simulations).

$$
\left.\begin{array}{r}
E_{1}=a+b \\
E_{2}=a e^{j \varphi}+b e^{-j \varphi} \\
E_{3}=a e^{2 j \varphi}+b e^{-2 j \varphi}
\end{array}\right\} \rightarrow \frac{b}{a}=\frac{\sqrt{u^{2}-4}-v}{\sqrt{u^{2}-4}+v},
$$

where $a$ and $b$ are the amplitudes of forward wave and backward wave as seen in Fig. $14 ; E_{1}, E_{2}$ and $E_{3}$ are the peak electrical field (complex number) of the last three cells; $\varphi$ are the phase advance $(2 / 3 \pi)$ between the two cells; and $u=\frac{E_{3}+E_{1}}{E_{2}}, v=\frac{E_{3}-E_{1}}{E_{2}}$. The reflection due to the mismatch in the output coupler is $\frac{b}{a}$.
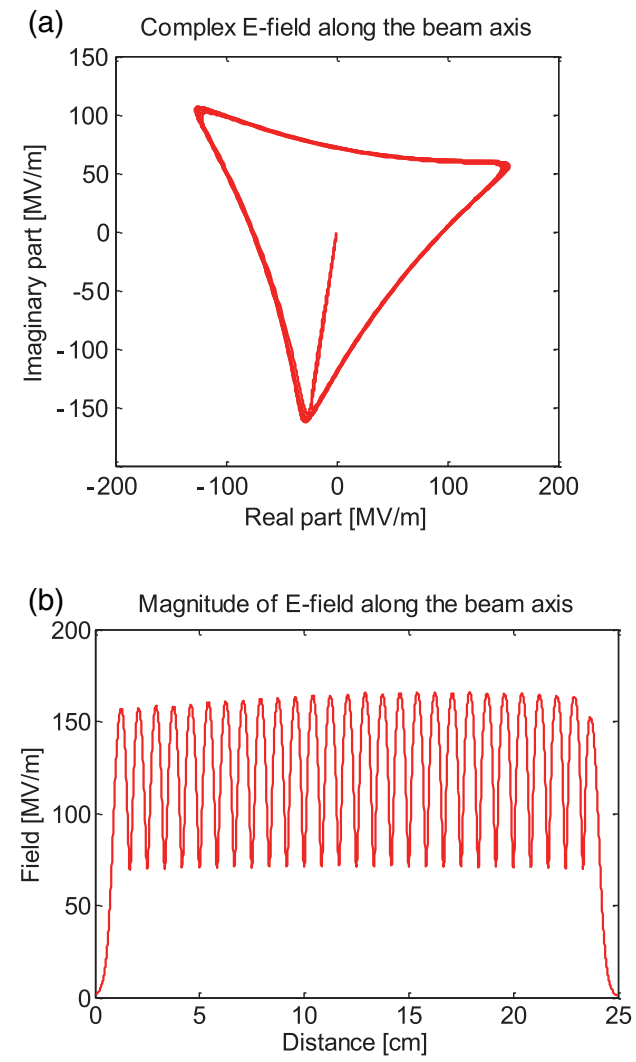

FIG. 16. Electrical accelerating field distribution on the beam axis of the new CLIC-G tapered structure: (a) in the complex plane; (b) magnitude.
TABLE I. Structure parameters of CLIC-G* design. The parameters of CLIC-G inside the braces are given in [2], outside are recalculated in latest HFSS version.

\begin{tabular}{lcc}
\hline \hline & CLIC-G & CLIC-G* \\
\hline Rounding $[\mathrm{mm}]$ & 0.5 & 1.0 \\
Manufacturing cost reduction & $\ldots$ & $7 \%$ \\
Shunt impedance $[\mathrm{M} \Omega / \mathrm{m}]$ & 92.0 & 95.4 \\
Peak input power $[\mathrm{MW}]$ & 63.5 & 62.3 \\
rf to beam efficiency & $27.8 \%$ & $28.4 \%$ \\
Filling time [ns] & 67 & 66 \\
Maximum electric field $[\mathrm{MV} / \mathrm{m}]$ & $239(230)$ & 235 \\
Maximum $S c\left[\mathrm{MW} / \mathrm{mm}^{2}\right]$ & 5.65 & 5.54 \\
Maximum temperature rise $[\mathrm{K}]$ & $50(46)$ & 40 \\
\hline \hline
\end{tabular}

The reflection due to the mismatch in the input coupler could be directly exanimated by the port reflection coefficiency $S_{11}$ in the coupling waveguides if the downstream of the structure is good matched. Dimensions of both output and input coupler cells were carefully tuned in HFSS simulations [31]. The reflection of input coupler is less than $-50 \mathrm{~dB}$ as seen in Fig. 15. According to Eq. (1), the
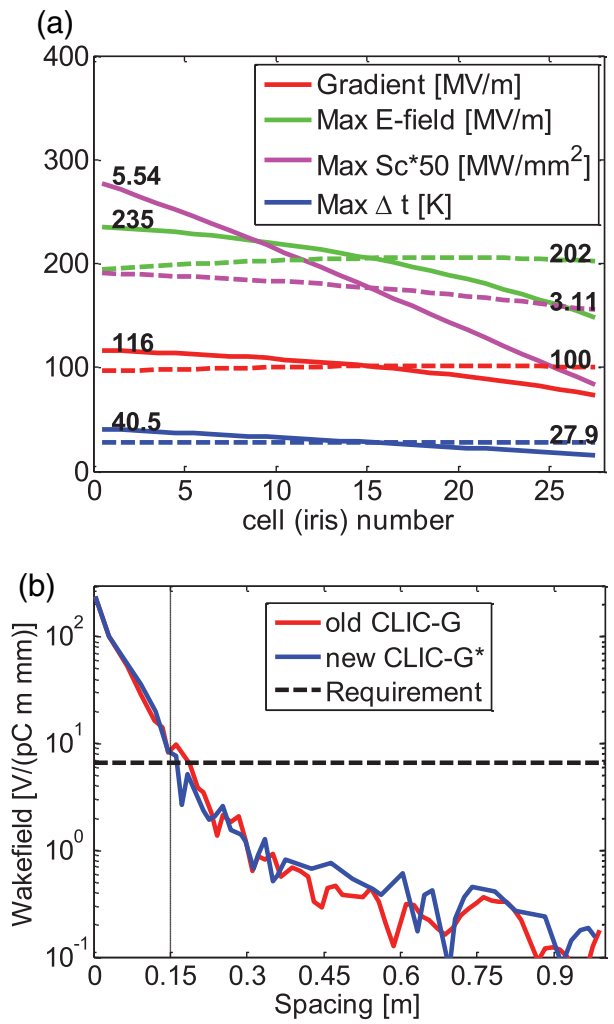

FIG. 17. (a) Distribution of $\mathrm{rf}$ parameters (red: accelerating gradient; blue: pulse temperature rise; green: maximum surface electrical field; magenta: maximum modified pointing vector [30]; dashed lines for unloaded $100 \mathrm{MV} / \mathrm{m}$ and solid lines for loaded $100 \mathrm{MV} / \mathrm{m}$ ) along the CLIC-G* structure cells. (b) Simulation results of transverse wakefield in the old baseline CLIC-G and new CLIC-G* structures (enveloped value). 
backward reflection at the output coupler was calculated by field distribution shown in Fig. 15 and is also less than $-50 \mathrm{~dB}$. Figure 16 shows a flat field distribution which indicates that the structure geometry is already well tuned.

\section{Parameters of the new structure design}

The newly optimized design integrates all the features introduced above and was named "CLIC-G $*$." The serial alphabet " $G$ " keeps the same since the new design does not change the iris parameters. The detailed parameters are listed in Table I. Distributions of rf parameters on cells were calculated by the algorithm developed in [32] and are plotted in Fig. 17(a).

Compared to the old CLIC-G design, this new design reduces the temperature rise from 50 to $40 \mathrm{~K}$. The estimated cost is reduced by $7 \%$ and the power consumption is $1 \mathrm{MW}$ lower due to the higher shunt impedance of structure cells. The simulated wakefield (enveloped value) of the new design is shown in Fig. 17(b) and is compared to the CLIC-G design from the CDR [3]. The attenuation of two curves is similar to each other. The wakefield potential of the new design at the positon of the second bunch $(0.15 \mathrm{~m})$ is $2 \mathrm{~V} / \mathrm{pC} / \mathrm{m} / \mathrm{mm}$, as required by beam dynamics.

\section{HOM DAMPING LOAD DESIGN}

The beam dynamics study for the CLIC main linac indicates that the transverse wakefield kick of a bunch on the following bunch must be suppressed to less than $6.6 \mathrm{~V} / \mathrm{pC} / \mathrm{m} / \mathrm{mm}$, in order to maintain the beam stability in the main linac $[33,34]$. The peak value of the excited transverse wakefield is about $250 \mathrm{~V} / \mathrm{pC} / \mathrm{m} / \mathrm{mm}$. Therefore the wakefield kick should be suppressed by nearly 2 orders of magnitude between one bunch separation $(0.5 \mathrm{~ns})$. In order to meet this criteria, HOM damping loads in the structure cells should provide strong absorption to all major dipole modes with their frequencies
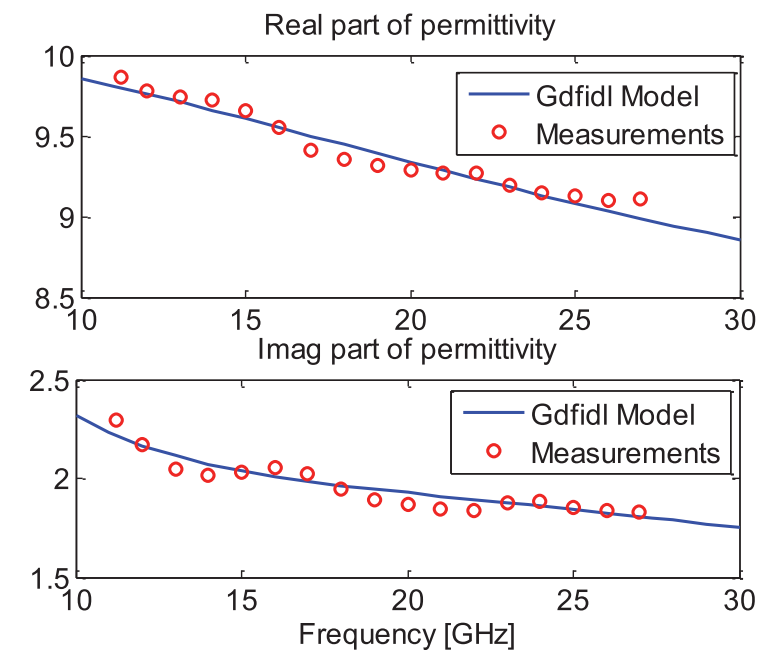

FIG. 18. Material properties of the HOM damping load.

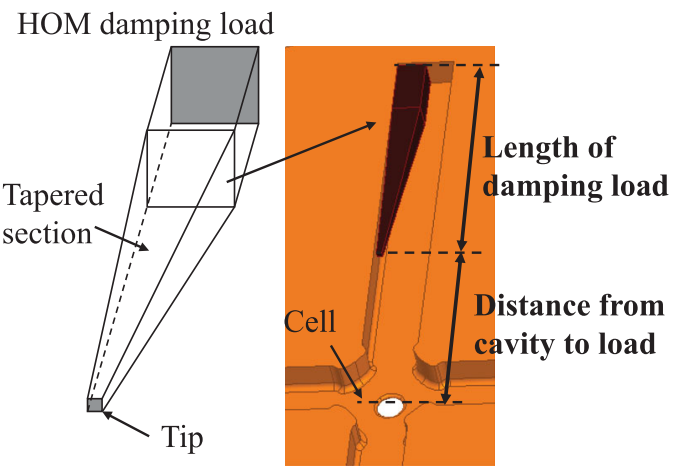

FIG. 19. Geometry of a HOMs damping load.

ranged from 15 to $45 \mathrm{GHz}$. The design of HOM damping loads was verified by the GDFIDL simulations on a CLIC-G* structure assembled together with these loads.

A silicon carbide material named "EkaSiC-P" is used as a reference for the design of the damping load. The measured data of the permittivity and the loss tangent of "EkaSiC-P" are shown in Fig. 18 [35]. Parametrized data on the permittivity and the dielectric loss tangent for this material in the GDFIDL simulations are also shown. The geometry of loads was designed as a smooth tapered box as shown in Fig. 19. The tip in the geometry has a small crosssection area in order to reduce the reflection from the tip and the design of the smooth tapered section is to achieve strong and broadband absorption.

HOM loads are placed in the damping waveguides of the CLIC accelerating structure cell. Though the frequency of the fundamental working mode is under cutoff in the waveguides, there is still a small fraction of the fundamental mode rf power penetrating in the waveguide and which is then absorbed by loads. Since the field of the working mode is exponentially decaying versus the depth in the waveguide, a certain distance from the load to the central cavity is needed to reduce power dissipation in loads

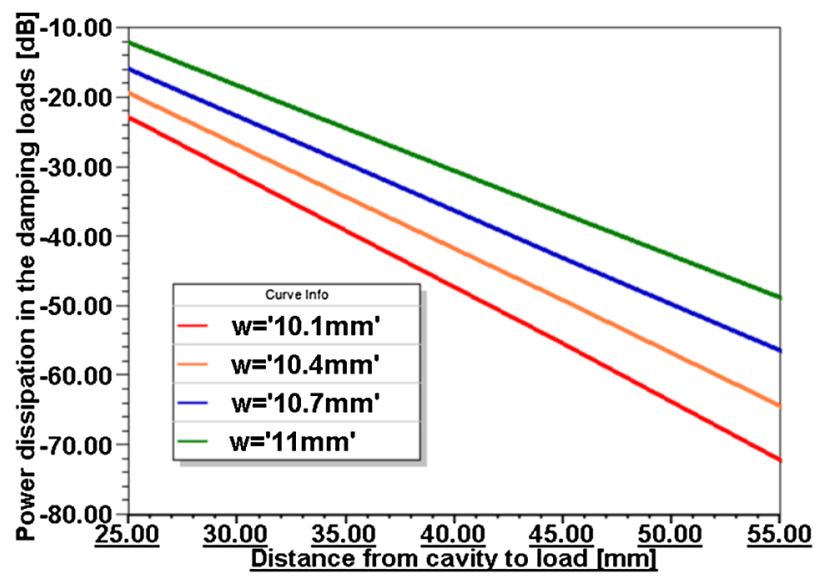

FIG. 20. Power dissipation in the damping load of working frequency ( $0 \mathrm{~dB}=$ power dissipation in the cell wall) versus the distance from the cavity central to the load and the waveguide width. 
as shown in Figs. 19 and 20. In the baseline CLIC-G design, this distance is $5 \mathrm{~cm}$ and the length of the load is $4 \mathrm{~cm}$. The overall cell diameter is $20 \mathrm{~cm}$. The estimated peak absorbed power of the working mode at one load is about $50 \mathrm{~W}$. The average power dissipation at one HOM load is $0.6 \mathrm{~mW}$ for $50 \mathrm{~Hz}$ repetition rate and $240 \mathrm{~ns}$ pulse width.

The new CLIC-G* accelerating structure design has a narrower waveguide width $(10.1 \mathrm{~mm})$ than the baseline design $(11 \mathrm{~mm})$. The distance between damping loads and the cavity of the new design can be shorter since the decay of the working mode is stronger. The geometry of the HOM load is reoptimized to fit the beam dynamic requirements. As shown in Fig. 21(a), the length of the HOM load for the new design is $4.4 \mathrm{~cm}$, a bit longer than the baseline design. This is because that length of smooth tapered section needs to be matched with the propagating wavelength of the HOM, which becomes longer for a smaller waveguide width.

The overall cell diameter of the new design is $17 \mathrm{~cm}$, shorter than the old CLIC-G design $(20 \mathrm{~cm})$ but still rather large for manufacturing. Two more compact designs as shown in Figs. 21(b) and 21(c) are proposed in order to reduce the manufacturing cost. These designs used shorter damping loads, which cause significant reflection of HOMs and weaken the wakefield suppression. The reflection can be compensated by changing the geometry in the damping waveguide: either added matching step on the side wall of the waveguide or made bended waveguides. Another potential advantage for the bended waveguides is reducing the amounts of manifolds (for vacuum pumping) from four to only two. Both matching ways could achieve the compact cell design and the dimensions are shown in Fig. 18.
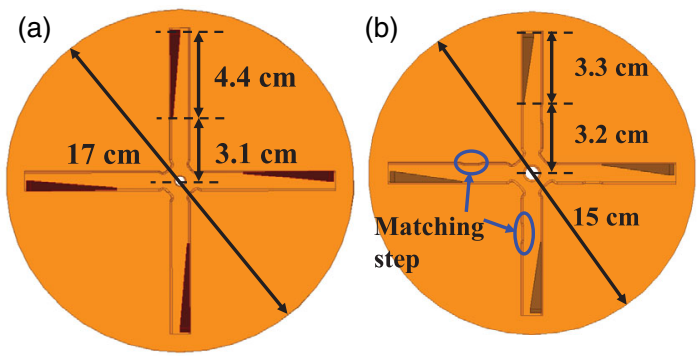

(c)

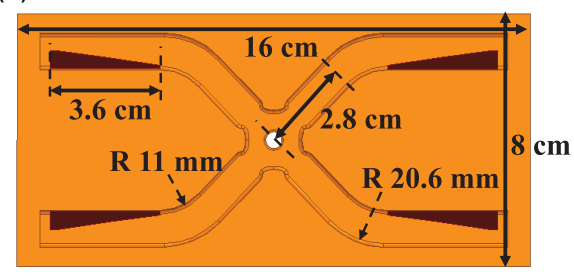

FIG. 21. Three designs of damping loads for new CLIC-G structure: (a) regular waveguides; (b) waveguides with matching step; (c) bended waveguides.
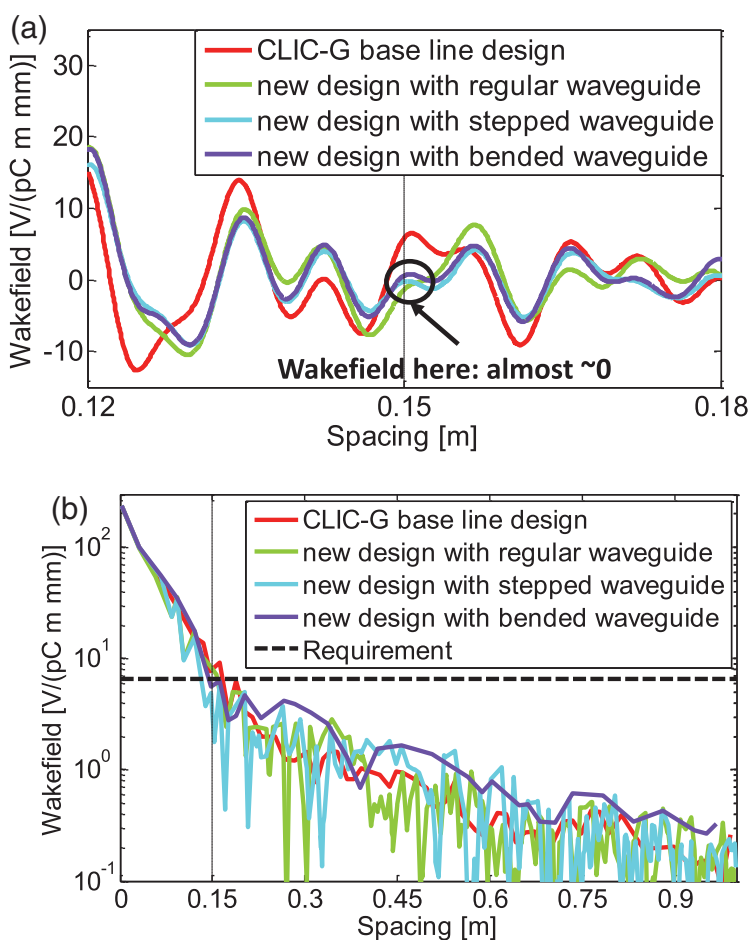

FIG. 22. Wakefield of all designs in two difference scales: (a) wakefield at the nearby position of the second bunch; (b) logscale enveloped wakefield plot from 0 to 1 meter.

The wakefield results for three designs are shown in Fig. 21. Two different scales are plotted. The plot in Fig. 22(a) is the wakefield value at the position of the second following bunch $(0.15 \mathrm{~m})$. Due to the cancellation of the reflections from loads and from the waveguide, the transverse wakefield kick amplitude at the position of the second bunch for the new structure design with all three HOM damping load schemes is smaller than that of the old CLIC-G design.

Three factors $F_{c}, F_{\text {rms }}$ and $F_{\text {worst }}$ are listed in Table II to state the overall effect of transverse wakefield on the beam bunches for all designs. These factors give the amplification of transverse beam jitter in the main linac due to the long range transverse wakefield in three different ways and details are introduced in [34]. Given by the numbers listed in Table II, the new CLIC-G* structure and corresponding HOM damping load designs show better overall wakefield

TABLE II. Transverse beam jitter amplification factors in wakefield suppression of all structure designs.

\begin{tabular}{lccr}
\hline \hline Design & $F_{c}$ & $F_{\text {rms }}$ & $F_{\text {worst }}$ \\
\hline Old baseline CLIC-G & 1.07 & 3.9 & 15.6 \\
CLIC-G* with regular waveguides & 1.02 & 1.1 & 2.9 \\
CLIC-G* with stepped waveguides & 1.02 & 1.2 & 2.7 \\
CLIC-G* with bended waveguides & 1.03 & 1.5 & 6.3 \\
Beam dynamics requirement & & $<5$ & \\
\hline \hline
\end{tabular}




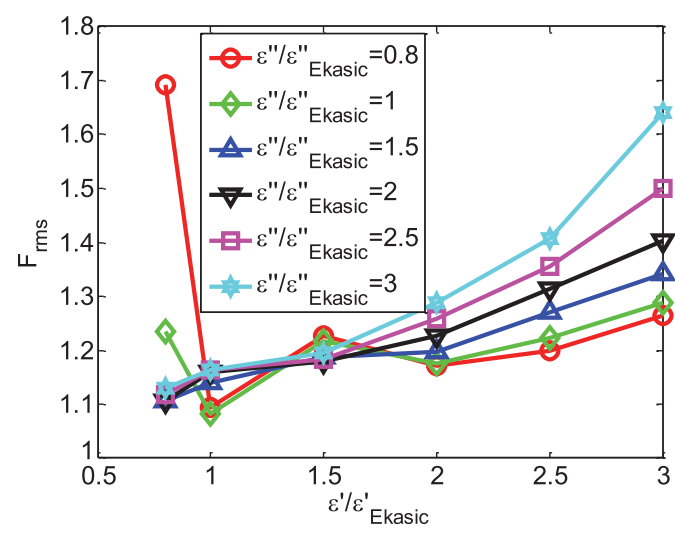

FIG. 23. Wakefield suppression for rf loads $\left(F_{\text {rms }}\right)$ versus various material properties $\left(\varepsilon^{\prime}\right.$ : real part of permittivity; $\varepsilon^{\prime \prime}$ : imaginary part of permittivity).

suppression than that of the old baseline design, which can increase the luminosity.

It is necessary to check the dependence of wakefield suppression on variation of the permittivity and the loss tangent of the $\mathrm{SiC}$ material, since material properties may vary from one manufacturing batch to another and measurements on material properties may be not accurate. Wakefield simulation of new CLIC-G structure (regular waveguide) with the HOM loads with same geometries but various material properties were carried out. The simulation swept the real $\varepsilon^{\prime}$ and imaginary part $\varepsilon^{\prime \prime}$ of the damping material in the range from $80 \%$ to $300 \%$ of the original value and properties of many silicon carbide materials used for $\mathrm{rf}$ absorber are in this range [36-38]. As shown in Fig. 23, the simulated wakefield suppression of all points is far better than the requirements $\left(F_{\text {rms }}<5\right)$. These simulation results prove that the design of this geometry for the damping loads in Fig. 19 is applicable for a quite large range of the material properties.

\section{HOM-FREE INPUT POWER COUPLER}

The geometry of input and output coupler cells is similar to those of regular cells (see Fig. 13). Unlike regular cells and output coupler cell, two power coupling waveguide branches of the input coupler cell are not terminated by HOM damping loads. As shown in Fig. 24, a power splitter is connected to the coupling waveguides for the doublefeeding coupler scheme. The excited dipolar wakefield in the input coupler will transmit through the coupling waveguide branches and will reach the power splitter. Figure 25(a) shows the simulated transverse wakefield in two cases: the power splitter fully absorbs all HOMs or reflects all HOMs. The discrepancy of wakefields in these two cases shows the amplitude of reflected transverse wakefield at the power splitter is at the level of $3 \mathrm{~V} /(\mathrm{pC} \mathrm{mmm})$. It causes a non-negligible increase in $F$-factors as shown in Fig. 25(b). The suppression of this

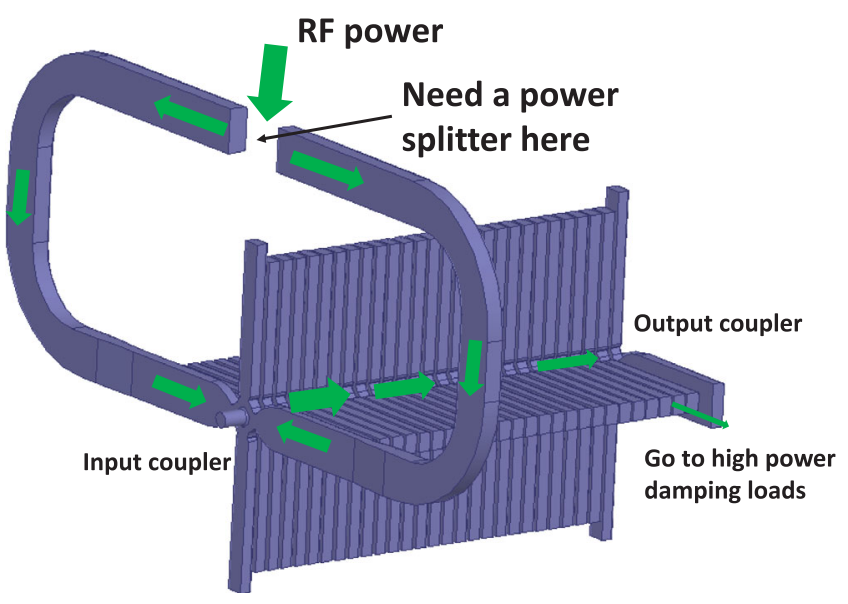

FIG. 24. Power coupling circuit of CLIC accelerating structure.

reflected wakefield is needed. A major dipole mode with the frequency of $17 \mathrm{GHz}$ dominates the reflected wakefield, as shown in the wakefield spectrum in Fig. 25(b). Consequently, a special design of the power splitter is needed to reduce its reflection of $17 \mathrm{GHz}$.

As shown in Fig. 26, the power splitter should split the working frequency to two waveguide branches at the same phases and absorb dipolar wakefields from two branches with 180-degree phase differences. Magic-T is the perfect solution to this requirement and such a design can suppress the unwanted HOMs and is named HOMagic-T. Ports \#2
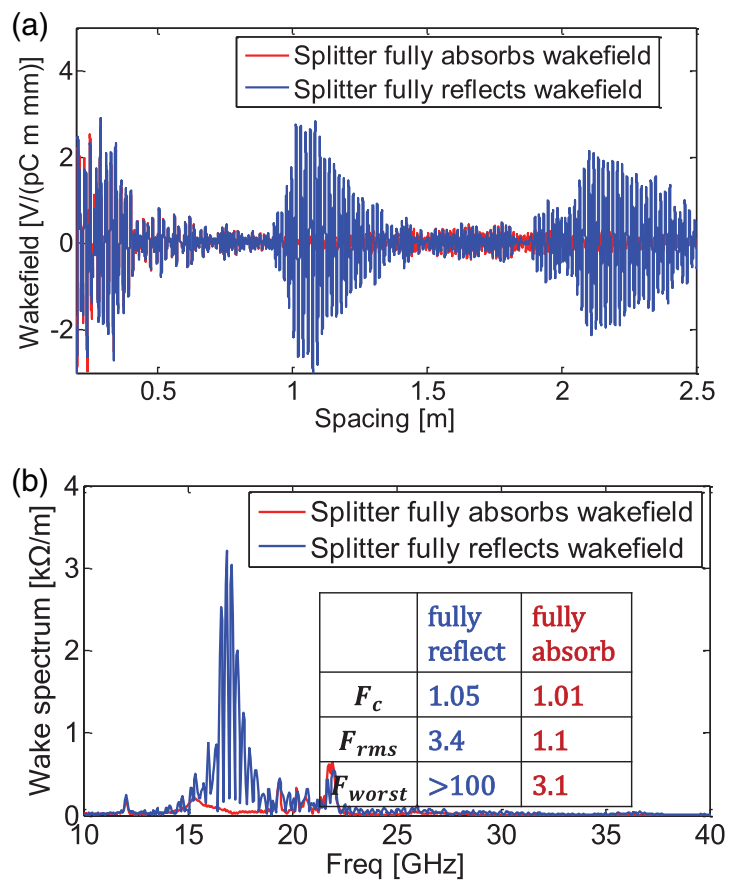

FIG. 25. Transverse wakefield of new CLIC accelerating structure with splitter absorbing or reflecting the dipolar wakefield: (a) time domain (please note the horizontal scale starts not from 0); (b) spectrum. 

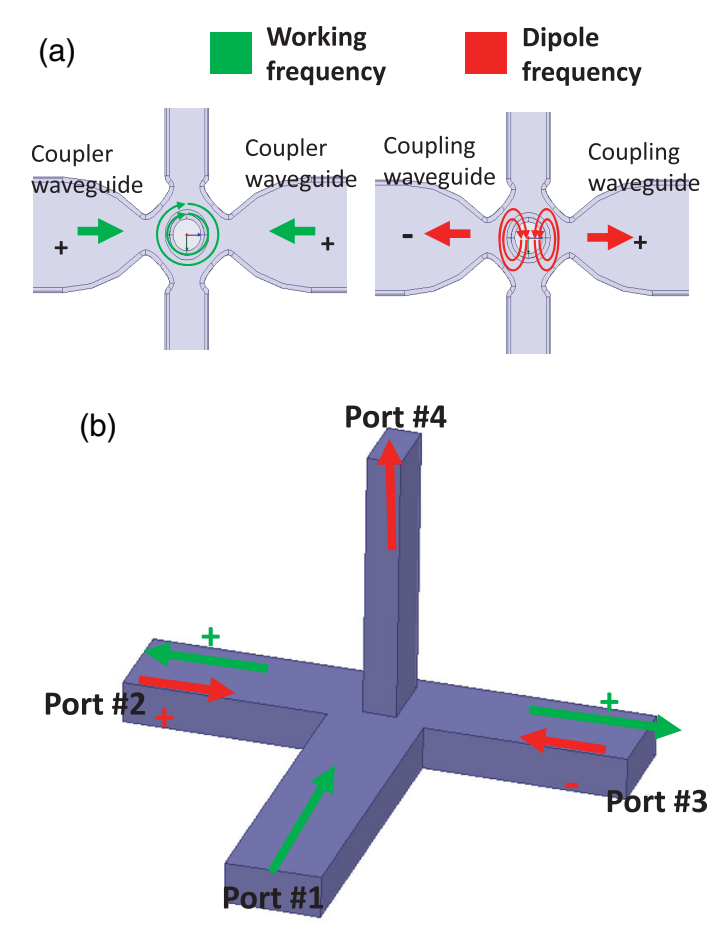

FIG. 26. Power coupling and HOM damping in (a) the coupler cell and (b) the HOMagic-T.

and \#3 of the HOMagic-T are connected to two waveguide branches of the input coupler cell, port \#1 is connected to the power source and port \#4 is terminated by a HOM damping load to damp the dipolar wakefields.

The design of this HOMagic-T is difficult because the WR90 waveguide which is used here for transmitting $\mathrm{X}$-band $\mathrm{rf}$ power allows multiple waveguide modes $\left(\mathrm{TE}_{10}\right.$, $\left.\mathrm{TE}_{20}, \mathrm{TE}_{01}, \mathrm{TE}_{11}\right)$ of the major dipole band $(17 \mathrm{GHz})$ to propagate. The final geometry design is shown in Fig. 27 after careful optimization. A step geometry in the central part of the Magic-T is used to reduce the mismatch of

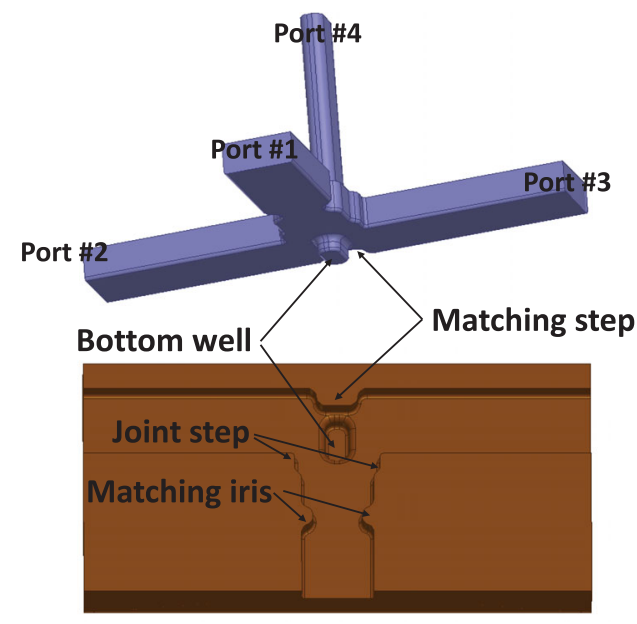

FIG. 27. Geometry of the special HOMagic-T design (top: view of vacuum part; bottom: view of metal part).
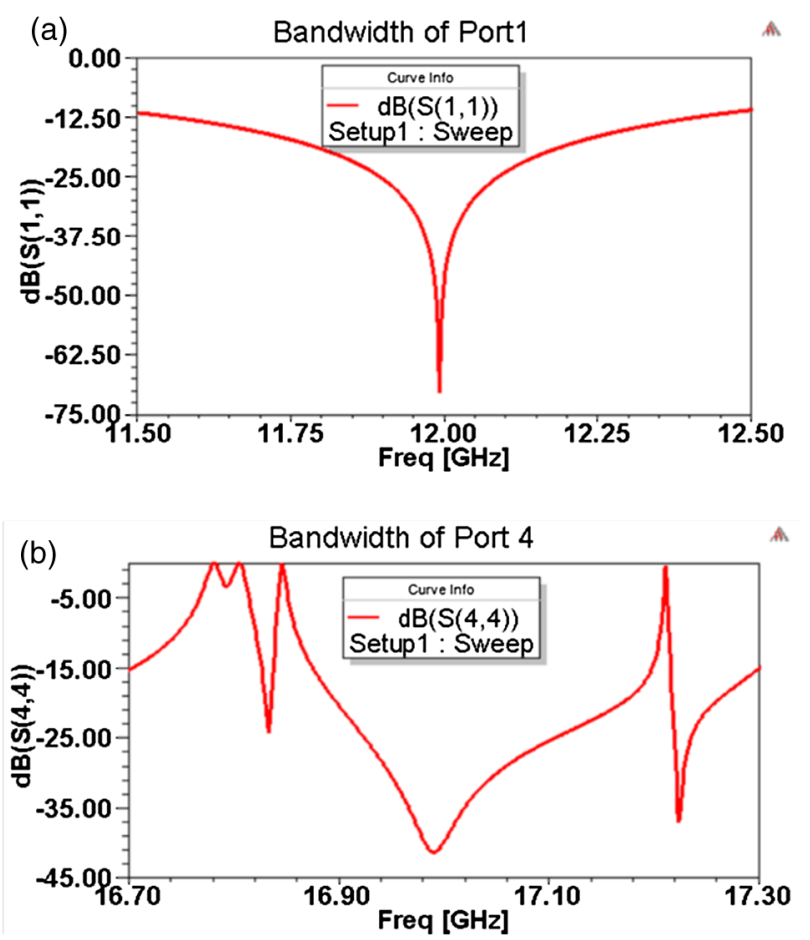

FIG. 28. Bandwidth of (a) the working frequency and (b) the major dipole frequency in the Magic-T design.
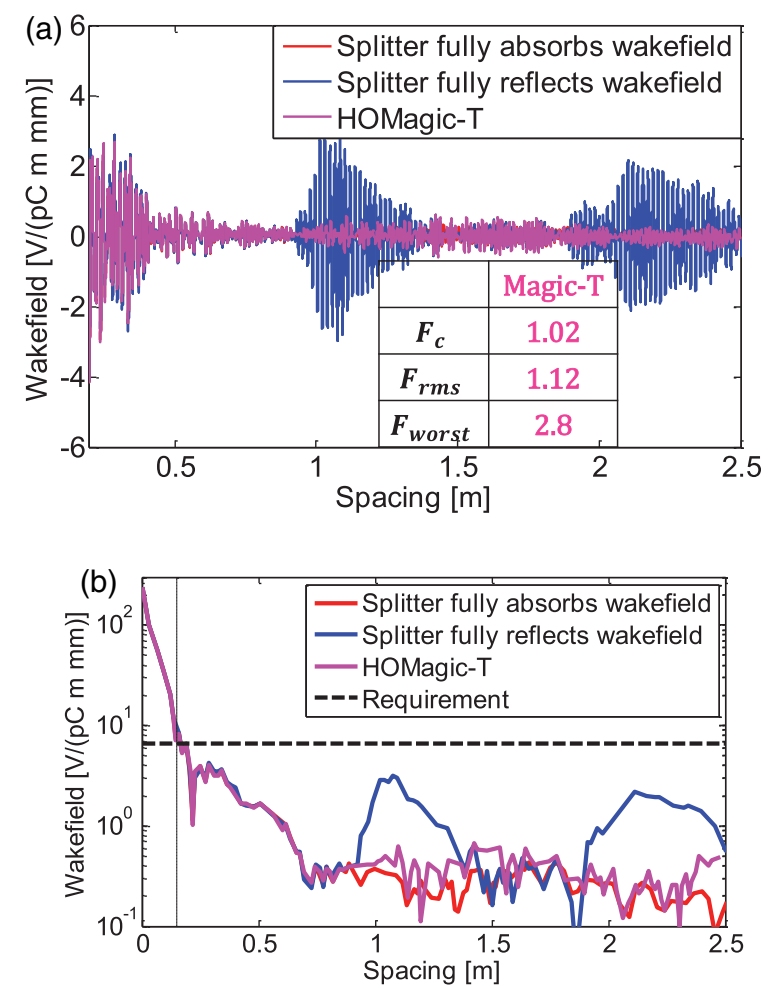

FIG. 29. Wakefield of the new CLIC-G accelerating structure with the HOMagic-T design: (a) linear scale; (b) enveloped value in $\log$ scale. 
splitting the working frequency, which can improve the bandwidth of working frequency as well as reduce the maximum surface field (peak surface electrical field $=$ $37 \mathrm{MV} / \mathrm{m}$ for transmitting $62 \mathrm{MW}$ power from port \#1). There is a well-like shape in the bottom part of the Magic-T, which is used to keep the symmetry and suppress the $\mathrm{TE}_{02}$ like waveguide propagating mode of the major dipole band. The step located between Magic-T and branch 1\# is used to tune the frequency of trapped modes away from the major dipole band. The matching iris in the branch \#1 is used to make a perfect match for working frequency. Figure 28 shows the HFSS simulation results, where subfigure (a) shows the bandwidth of working frequency and subfigure (b) shows the reflection of major dipole band.

Wakefield simulation results of the new CLIC-G structure with input coupler connecting this HOMagic-T design is shown in Fig. 29 and are compared to those of the input coupler terminated by prefect matched layer. Three $F$-factors are also listed in the figures [compare to Fig. 25(b)] and verified that this HOMagic-T design fit the wakefield suppression requirements.

\section{CONCLUSION}

Recent research on the CLIC-G baseline structure including high power tests, wakefield measurements and manufacturing studies has given the direction for further optimization on the CLIC-G structure geometry. A fourth order polynomial shape was proposed for the wall profile of new CLIC-G structure cells instead of elliptical shape to reduce the surface magnetic field. We increased the rounding of structure cells from 0.5 to $1 \mathrm{~mm}$, in order to reduce the total manufacturing cost. With the $1 \mathrm{~mm}$ rounding, we reoptimized the waveguide geometry (new waveguide width: $10.1 \mathrm{~mm}$ ) to reduce the surface magnetic field. The waveguide geometry (opening) is set individually for each structure cell to avoid the appearance of hot cells in terms of pulse temperature rise. With all of the above improvements on the geometry employed, $10 \mathrm{~K}$ reduction of maximum pulse temperature rise (allow 10\% gradient increasing if the pulse heating is the limit of achieving higher gradient), $7 \%$ reduction of the manufacturing cost and $1 \mathrm{MW}$ reduction of the input power are achieved for the new CLIC-G structure design.

The geometry of HOM damped load for the new CLIC-G structure design was optimized to reduce transverse size of the structure. Other than a uniform damping waveguide, a new waveguide geometry with matching step and bended waveguide was proposed to further reduce the diameter. Wakefield suppression of the three proposed HOM load designs is verified by GDFIDL simulations and the results are even better than the old CLIC-G baseline design.

A special power splitter using HOMagic-T geometry was designed for the double feed input coupler. This power splitter could absorb the major dipole band (17 GHz) excited in the coupler cell and meet the wakefield suppression requirements in the new CLIC-G design.

\section{ACKNOWLEDGMENTS}

The authors are grateful to W. Wuensch for his constant support, useful comments and careful reading of the manuscript.

[1] R. Tomás, Overview of the Compact Linear Collider, Phys. Rev. ST Accel. Beams 13, 014801 (2010).

[2] CLIC Conceptual Design Report (CDR), 2012, http://projectclic-cdr.web.cern.ch/project-CLICCDR/CDR_Volume1.pdf.

[3] A. Grudiev and W. Wuensch, Design of the CLIC main linac accelerating structure for CLIC Conceptual Design Report, in Proceedings of the 25th International Linear Accelerator Conference LINAC10, Tsukuba, Japan (KEK, Tsukuba, Japan, 2010).

[4] H. Zha, J. Shi, H. Chen, A. Grudiev, W. Wuensch, C. Tang, and W. Huang, Choke-mode damped structure design of the Compact Linear Collider main linac, Phys. Rev. ST Accel. Beams 15, 122003 (2012).

[5] V.F. Khan, A. D'Elia, R. M. Jones, A. Grudiev, W. Wuensch, G. Riddone, and V. Soldatov, Wakefield and surface electromagnetic field optimization of manifold damped accelerating structures for CLIC, Nucl. Instrum. Methods Phys. Res., Sect. A 657, 131 (2011).

[6] FACET facility in SLAC National Laboratory, http://portal .slac.stanford.edu/sites/ard_public/facet/Pages/default.aspx.

[7] GDFIDL, http://www.gdfidl.de.

[8] H. Zha, A. Latina, A. Grudiev, G. De Michele, A. Solodko, W. Wuensch, D. Schulte, E. Adli, N. Lipkowitz, and G. S. Yocky, Beam-based measurements of long range transverse wakefields in CLIC main linac accelerating structure, Phys. Rev. Accel. Beams 19, 011001 (2016).

[9] A. Degiovanni, W. Wuensch, and J. G. Navarro, Comparison of the conditioning of high gradient accelerating structures, Phys. Rev. Accel. Beams 19, 032001 (2016).

[10] T. Higo, T. Abe, Y. Arakida, Y. Higashi, S. Matsumoto, T. Shidara, T. Takatomi, M. Yamanaka, A. Grudiev, G. Riddone et al., Comparison of high gradient performance in varying cavity geometries, in Proceedings of the 4th International Particle Accelerator Conference, IPAC2013, Shanghai, China, 2013 (JACoW, Shanghai, China, 2013), pp. 2741-2743.

[11] J. Q. Luis Navarro, R. Corsini, A. Degiovanni, S. Döbert, A. Grudiev, O. Kononenko, G. McMonagle, S. Rey, S. Anastasiya, and I. Syratchev, Effect of beam-loading on the breakdown rate of high gradient accelerating structures, in 27th Linear Accelerator Conference, Geneva, Switzerland, 2014 (JACoW, Geneva, 2014), p. TUPP033.

[12] A. Degiovanni et al., Diagnostics and analysis techniques for high power X-band accelerating structures, in 27th Linear Accelerator Conference, Geneva, Switzerland, 2014 (JACoW, Geneva, 2014), p. TUPP029.

[13] N. Shipman et al., Experimental study of DC vacuum breakdown and application to high-gradient accelerating 
structures for CLIC, Reports No. CERN-THESIS-2014278 and No. CERN-ACC-2016-0014, 2016, p. 196.

[14] N. Catalan-Lasheras, A. Degiovanni, S. Doebert, W. Farabolini, J. Kovermann, G. McMonagle, S. Rey, I. Syratchev, L. Timeo, W. Wuensch, B. Woolley, and J. Tagg, Experience operating an X-band high-power test stand at CERN, in Proceedings of International Particle Accelerator Conference IPAC14 (JACoW, Dresden, 2013), pp. 2288-2290.

[15] A. Degiovanni, S. Doebert, W. Farabolini, A. Grudiev, J. Kovermann, E. Montesinos, G. Riddone, I. Syratchev, R. Wegner, W. Wuensch, A. Solodko, and B. Woolley, Highgradient test results from a CLIC prototype accelerating structure: TD26CC, in Proceedings of the International Particle Accelerator Conference IPACI4 (JACoW, Dresden, 2013), pp. 2285-2287.

[16] W. Wuensch, Advances in the understanding of the physical processes of vacuum breakdown, Reports No. CERNOPEN-2014-028 and No. CLIC-Note-1025, 2013, p. 18.

[17] S. Matsumoto, T. Abe, Y. Higashi, T. Higo, and Y. Du, High gradient test at Nextef and high-power long-term operation of devices, Nucl. Instrum. Methods Phys. Res., Sect. A 657, 160 (2011).

[18] W. Wuensch, High-gradient acceleration: CLIC and beyond, CLIC workshop 2016, Geneva, Switzerland (2016), https://indico.cern.ch/event/449801/.

[19] S. Atieh (private communication).

[20] L. Laurent, S. Tantawi, V. Dolgashev, C. Nantista, Y. Higashi, M. Aicheler, S. Heikkinen, and W. Wuensch, Experimental study of rf pulsed heating, Phys. Rev. ST Accel. Beams 14, 041001 (2011).

[21] F. Wang, C. Adolphsen, and C. Nantista, Performance limiting effects in X-band accelerators, Phys. Rev. ST Accel. Beams 14, 010401 (2011).

[22] H. H. Braun, S. Döbert, I. Wilson, and W. Wuensch, Frequency and Temperature Dependence of Electrical Breakdown at 21, 30, and $39 \mathrm{GHz}$, Phys. Rev. Lett. 90, 224801 (2003).

[23] A. T. Perez Fontenla, TD24 post-mortem SEM analysis, at the CLIC rf development meeting (2014), https://indico .cern.ch/event/320252/.

[24] D. P. Pritzkau, RF pulsed heating, Technical Report No. SLAC-R-577, 2001.

[25] O. Kononenko and A. Grudiev, Transient beamloading model and compensation in Compact Linear
Collider main linac, Phys. Rev. ST Accel. Beams 14, 111001 (2011).

[26] R. M. Jones, V. A. Dolgashev, and J. W. Wang, Dispersion and energy compensation in high-gradient linacs for lepton colliders, Phys. Rev. ST Accel. Beams 12, 051001 (2009).

[27] HFSS, http://www.ansys.com.

[28] A. Grudiev, Update on the optimization procedure, inputs and results, presentation of the CLIC reference structure, 2nd CLIC Advisory Committee, Geneva, Switzerland (2008), https://indico.cern.ch/event/24998/.

[29] A. Grudiev, Very short-range wakefields in strongly tapered disk-loaded waveguide structures, Phys. Rev. ST Accel. Beams 15, 121001 (2012).

[30] A. Grudiev, S. Calatroni, and W. Wuensch. New local field quantity describing the high gradient limit of accelerating structures. Phys. Rev. ST Accel. Beams 12, 102001 (2009).

[31] J. Shi, A. Grudiev, and W. Wuensch, Tuning of X-band traveling-wave accelerating structures, Nucl. Instrum. Methods Phys. Res., Sect. A 704, 14 (2013).

[32] A. Lunin, V. Yakovlev, and A. Grudiev, Analytical solutions for transient and steady state beam loading in arbitrary traveling wave accelerating structures, Phys. Rev. ST Accel. Beams 14, 052001 (2011).

[33] R. M. Jones, Wakefield suppression in high gradient linacs for lepton linear colliders, Phys. Rev. ST Accel. Beams 12, 104801 (2009).

[34] D. Schulte, Multibunch calculations in the CLIC main linac, in Proceedings of the 23rd Particle Accelerator Conference, Vancouver, Canada, 2009 (IEEE, Piscataway, NJ, 2009).

[35] G. De Michele, Wakefield simulations and measurements for the CLIC rf accelerating structure, Ph.D. thesis, École Polytechnique Fédérale de Lausanne, 2014.

[36] R. Eichhorn et al., Higher order mode absorbers for high current ERL applications, in Proceedings of SRF2015, Whistler, BC, Canada (2015), p. THBA05.

[37] F. Marhauser et al. Absorber materials at room and cryogenic temperatures, in Proceedings of IPAC2011, San Sebastián, Spain (EPS-AG, Spain, 2011), p. TUPS106.

[38] A. Degiovanni et al. RF dielectric properties of $\mathrm{SiC}$ ceramics and their application to design of HOM absorbers, in Proceedings of the 21st Particle Accelerator Conference, Knoxville, 2005 (IEEE, Piscataway, NJ, 2005), p. WPAT010. 